\title{
SOME FUNCTIONS WITH A UNIQUE INVARIANT MEAN
}

\author{
MICHEL TALAGRAND ${ }^{1}$
}

\begin{abstract}
In a large class of groups, we construct a function which has a unique invariant mean, but which is not Riemann-measurable.
\end{abstract}

1. Introduction and notations. Let $G$ be a compact group. For $f \in L^{\infty}=L^{\infty}(G)$ and $a \in G$, let $f_{a} \in L^{\infty}$ be given by $f_{a}(x)=f(a x)$ for $x \in G$. An invariant mean on $L^{\infty}$ is a positive linear functional $m$ such that $m(1)=1$ and $m\left(f_{a}\right)=m(f)$ for $f \in L^{\infty}, a \in G$. The normalized Haar measure $\lambda$ defines an invariant mean. We say $f \in L^{\infty}$ has a unique invariant mean if, for each invariant mean $m, m(f)=$ $\int f d \lambda$. A sufficient condition for $f$ to have a unique invariant mean is the following:

(1) For all $\varepsilon>0$, there exist $a_{1}, \ldots, a_{n}$ in $G$ with $\left\|(1 / n) \sum_{i=1}^{n} f_{a_{1}}-\int f d \lambda\right\|<\varepsilon$.

If $G$ is amenable as discrete, for example Abelian, it is known that this condition is also necessary.

It is clear that each continuous function has a unique invariant mean. An $f \in L^{\infty}$ will be said to be Riemann-measurable if it is (the class of) a function whose set of points of discontinuity is negligible. This is the same as saying that for all $\varepsilon>0$, there exist continuous functions $g_{1}<f<g_{2}$ with $\int\left(g_{2}-g_{1}\right) d \lambda<\varepsilon$. Then $f$ has a unique invariant mean. In fact, for each invariant mean $m$, we have $\int g_{1} d \lambda<m(f)<\int g_{2} d \lambda$ and both $\int g_{1} d \lambda$ and $\int g_{2} d \lambda$ differ from $\int f d \lambda$ by less than $\varepsilon$.

It is not hard to use (1) to construct in many groups functions $f$ which are not Riemann-measurable and have a unique invariant mean. A natural question, raised by L. A. Rubel and A. L. Shields [1, p. 39], is: Suppose for each continuous $g$, the function $f g$ has a unique invariant mean. Does it follow that $f$ is Riemann-measurable? We are going to give a negative answer to this question in a large class of groups.

2. Construction. The Haar measure of a measurable set $A \subset G$ will be denoted by $|A|$. If $A, B \subset G$, let $A B=\{a b: a \in A, b \in B\}$.

THEOREM. Suppose $G$ satisfies the following condition:

(2) $G$ is metrizable and there exist a sequence $\left(S_{n}\right)$ of measurable sets whose diameters go to zero and a sequence of finite sets $\left(P_{n}\right)$ of $G$ such that $S_{1}=G$, and

Received by the editors November 20, 1979 and, in revised form, May 14, 1980.

1980 Mathematics Subject Classification. Primary 28C10; Secondary 26A15.

Key words and phrases. Unique invariant mean, Riemann-measurable.

${ }^{1}$ This research was done while the author held a grant from NATO to visit Ohio State University. 
such that, for $n>1$, the sets $a S_{n+1}$ for $a \in P_{n+1}$ form a partition of $S_{n}$, and such that $Q_{n}=P_{2} \cdots P_{n}$ is a finite subgroup of $G$.

Then there exists a function $f: G \rightarrow\{0,1\}$, which is not Riemann-measurable, but such that fh has a unique invariant mean whenever $h$ is Riemann-measurable.

In fact, by an obvious argument, it would be enough to suppose that $G$ has a quotient which satisfies (2). It is also not hard to see that (2) is satisfied whenever $G=\mathbf{R} / \mathbf{Z}$ or $G=(\mathbf{Z} / 2 Z)^{\mathbf{N}}$. G.

Proof. Let $Q_{n}=P_{2} P_{3} \cdots P_{n}$. Hence the sets $a S_{n}$ for $a \in Q_{n}$ form a partition of

We shall construct by induction a sequence $X_{k}$ of subsets of $G$, and an increasing sequence of integers $n_{k}$ such that the following conditions hold:

$\left(A_{k}\right)$ There exists $A \subset Q_{n_{k}}$ such that $X_{k}=A S_{n_{k}}$.

$\left(B_{k}\right)$ For each $a \in Q_{k},\left|X_{k} \cap a S_{k}\right| \geqslant\left|S_{n_{k}}\right|,\left|\left(G \backslash X_{k}\right) \cap a S_{k}\right|>\left|S_{n_{k}}\right|$.

$\left(C_{k}\right)\left|X_{k} \Delta X_{k-1}\right|<2^{-k-2}\left|S_{n_{k-1}}\right|$ if $k>2$.

$\left(D_{k}\right) \forall r<k \forall a \in Q_{r}, \Sigma_{b \in Q_{n_{r}}} \chi_{X_{k} \cap a S_{r}}(b x)=$ constant.

Before we proceed to the construction, let us show why it implies the result. From the conditions $\left(C_{k}\right)$, it follows that there exists a measurable set $X \subset G$ with

$$
\left|X \Delta X_{k}\right|<\sum_{l>k}\left|X_{l} \Delta X_{l+1}\right|<\left|S_{n_{k}}\right| \sum_{l>k} 2^{-l-3}<\frac{1}{2}\left|S_{n_{k}}\right|
$$

The conditions $\left(B_{k}\right)$ show that for $a \in Q_{k},\left|X \cap a S_{k}\right|>0$ and $\left|(G \backslash X) \cap a S_{k}\right|>$ 0 . Since the diameter of $S_{k}$ goes to zero, each open set contains a set $a S_{k}$ for $k$ large and $a \in Q_{k}$. Hence $X$ and its complement meet each open set in a set of positive measure. It shows that $f=\chi_{X}$ is not Riemann-measurable.

Now if in condition $\left(D_{k}\right)$ we fix $r$ and let $k$ go to infinity, we get that for all $a \in Q_{r}$, we have

$$
\sum_{b \in Q_{m}} \chi_{a S_{r}}(b x) f(b x)=\text { constant }
$$

and hence from (1) the function $\chi_{a S_{r}} f$ has a unique invariant mean. Now, each continuous function $g$ can be uniformly approximated by a function of the type $\Sigma_{a \in Q_{p}} \alpha_{a} \chi_{a S_{p}}$ for $p$ large; hence $f g$ has a unique invariant mean. Now, if $h$ is Riemann-measurable, for each $\varepsilon>0$ there exist continuous functions $g_{1}, g_{2}$ with $g_{1}<h<g_{2}, \int\left(g_{2}-g_{1}\right) d \lambda \leqslant \varepsilon$, and hence, for each invariant mean $m, \int f g_{1} d \lambda<$ $m(f h)<\int f g_{2} d \lambda$ where $\int f\left(g_{2}-g_{1}\right) d \lambda<\varepsilon$, which shows that $f h$ has a unique invariant mean.

We start the construction by picking $a \in P_{2}$, and setting $n_{1}=2, X_{1}=a S_{n_{1}}$. Then $\left(A_{1}\right),\left(B_{1}\right),\left(D_{1}\right)$ are satisfied and $\left(C_{1}\right)$ is empty.

Now suppose the construction of $X_{k}$ has been done, such that the conditions $\left(A_{k}\right)$ to $\left(D_{k}\right)$ are satisfied. Enumerate $Q_{k+1}$ as $\left(a_{q}\right)_{1<q<l}$ where $l=$ card $Q_{k+1}$. The construction of $X_{k+1}$ will be done in $2 l$ steps. We are going to construct for $p<2 l$ sets $Y_{p}$ of $G$ and increasing integers $m_{p}$, with $Y_{1}=X_{k}, m_{1}=n_{k}$, such that the following conditions hold.

$\left(E_{p}\right)$ There exist $B \subset Q_{m_{p}}$ such that $Y_{p}=B S_{m_{p}}$. 
$\left(F_{p}\right)$ If $p=2 q$, then $\left|Y_{p} \cap a_{q} S_{k+1}\right|>\left|S_{m_{p}}\right|$. If $p=2 q-1$ then $\mid\left(G \backslash Y_{p}\right) \cap$ $a_{q} S_{k+1}|>| S_{m_{p}} \mid$.

$\left(G_{p}\right)\left|Y_{p} \Delta Y_{p-1}\right|<2^{-p-2-k}\left|S_{m_{p-1}}\right|$ if $p \geqslant 2$.

$\left(H_{p}\right) \forall r<k, \forall a \in Q_{r}, \Sigma_{b \in Q_{n_{r}}} \chi_{Y_{p} \cap a S_{r}}(b x)=$ constant.

Before we perform the construction, let us show that $X_{k+1}=Y_{2 l}$ satisfies $\left(A_{k+1}\right)$ to $\left(D_{k+1}\right)$ if we take $n_{k+1}=m_{2 l}$. First, $\left(A_{k+1}\right)$ is nothing else than $\left(E_{2 l}\right)$. Second, $\left(D_{k+1}\right)$ is the union of $\left(H_{2 l}\right)$ and the following (which is clearly true)

$$
\forall a \in Q_{k+1}, \sum_{b \in Q_{m_{t+1}}} \chi_{Y_{2 l} \cap a S_{k+1}}(b x)=\text { constant. }
$$

From the conditions $\left(G_{p}\right)$ we get that

$$
\left|X_{k} \Delta X_{k+1}\right|=\left|Y_{1} \Delta Y_{2 l}\right|<\sum_{p=2}^{2 l}\left|Y_{p} \Delta Y_{p-1}\right|<\sum_{r>2} 2^{-r-2-k}\left|S_{m_{1}}\right|<2^{-k-3}\left|S_{m_{k}}\right|
$$

and this is $\left(C_{k+1}\right)$. By the same argument we have $\left|Y_{p} \Delta X_{k+1}\right|<\frac{1}{2}\left|S_{m_{m}}\right|$ for all $p$. Now if $a \in Q_{k+1}$, let $q$ such that $a=a_{q}$. From $\left(F_{2 q}\right)$ we get

$$
\left|X_{k+1} \cap a S_{k+1}\right|>\left|Y_{2 q} \cap a_{q} S_{k+1}\right|-\left|Y_{2 q} \Delta X_{k+1}\right|>\frac{1}{2}\left|S_{m_{2 q}}\right|
$$

and hence from $\left(A_{k+1}\right),\left|X_{k+1} \cap a S_{k+1}\right| \geqslant\left|S_{n_{k+1}}\right|$. We get similarly $\mid\left(G \backslash X_{k+1}\right) \cap$ $a S_{k+1}|>| S_{n_{k+1}} \mid$ by considering $2 q-1$ instead of $2 q$. Suppose now that (for example, the case $2 q-1$ being similar) $Y_{2 q}$ and $m_{2 q}$ have been constructed satisfying $\left(E_{2 q}\right)$ to $\left(H_{2 q}\right)$. If $\left|\left(G \backslash Y_{2 q}\right) \cap a_{q+1} S_{k+1}\right|>0$ then this measure is greater than $\left|S_{m_{2 q}}\right|$ and it is enough to take $Y_{2 q+1}=Y_{2 q}, m_{2 q+1}=m_{2 q}$. If not, let $m_{2 q+1}$ be an integer such that

(3) $2^{2 q+4+k}$ card $Q_{k}\left|S_{m_{2 q+1}}\right|<\left|S_{m_{2 q}}\right|$.

Since $\left|Y_{1} \Delta Y_{2 q}\right|<\frac{1}{2}\left|S_{m_{1}}\right|$ it follows from $\left(B_{k}\right)$ that $\left|\left(G \backslash Y_{2 q}\right) \cap v S_{k}\right|>0$ for $v \in Q_{k}$. Take for $v$ the unique element in $Q_{k}$ such that $v S_{k} \supset a_{q+1} S_{k+1}$, i.e. such that $a_{q+1}=v w$ for a certain $w \in P_{k+1}$.

Set $Q^{\prime}=P_{k+2} P_{k+3} \cdots P_{m_{2 q}}, Q^{\prime \prime}=P_{m_{2 q}+1} P_{m_{2 q}+2} \cdots P_{m_{2 q+1}}$ and $S=S_{m_{2 q}}, S^{\prime}$ $=S_{m_{2 q+1}}$. Since $\left|\left(G \backslash Y_{2 q}\right) \cap v S_{k}\right|>0$, it follows from $\left(E_{2 q}\right)$ that there exist $w^{\prime} \in$ $P_{k+1}$ and $x \in Q^{\prime}$ with $v w^{\prime} x S \cap Y_{2 q}=\varnothing$. Since we have assumed $a_{q+1} S_{k+1} \subset Y_{2 q}$, we have vwxS $\subset Y_{2 q}$. Now let $y$ be any fixed element of $Q^{\prime \prime}$. We have $v w^{\prime} x y S^{\prime} \cap$ $Y_{2 q}=\varnothing, v w x y S^{\prime} \subset Y_{2 q}$. Each element $u \in Q=Q_{m_{2 q+1}}$ can be written in a unique way, $u_{1} u_{2} u_{3} u_{4}$, where $u_{1} \in Q_{k}, u_{2} \in P_{k+1}, u_{3} \in Q^{\prime}, \mu_{4} \in Q^{\prime \prime}$. Let $T$ be the bijection of $Q$ which lets $u$ be fixed if $u_{2} \notin\left\{w, w^{\prime}\right\}$ or $u_{3} \neq x$ or $u_{4} \neq y$ and which exchanges $u_{1} w^{\prime} x y$ and $u_{1} w x y$ for each $u_{1}$.

From $\left(E_{2 q}\right)$ we have $Y_{2 q}=B S$ for $B \subset Q_{m_{2 q}}$ and hence $Y_{2 q}=C S^{\prime}$ where $C=B Q^{\prime \prime}$. Define $Y_{2 q+1}=T(C) S^{\prime}$. It is obvious that $\left(E_{2 q+1}\right)$ is satisfied. Since $T$ moves at most 2 card $Q_{k}$ points of $Q$, we have

$$
\left|Y_{2 q} \Delta Y_{2 q+1}\right|<2 \operatorname{card} Q_{k}\left|S^{\prime}\right|<2^{-2 q-3-k}|S|
$$

from condition (3); that is, $\left(G_{2 q+1}\right)$ holds and $\left(F_{2 q+1}\right)$ follows from the fact that, since $v w^{\prime} x y S^{\prime} \cap Y_{2 q}=\varnothing$, we have $v w x y S^{\prime} \cap Y_{2 q+1}=\varnothing$ and $v w=a_{q+1}$. 
To complete the proof, it remains to show that $\left(H_{2 q+1}\right)$ holds. Fix $r<k$ and $a \in Q_{r}$, and define

$$
\alpha(x)=\sum_{b \in Q_{m_{r}}} \chi_{Y_{2 q+1} \cap a S_{r}}(b x)=\sum_{\substack{b \in Q_{n^{\prime}} \\ t \in T(C)}} \chi_{t S^{\prime} \cap a S_{r}}(b x) .
$$

We have $S_{r}=R S^{\prime}$, where $R=P_{r+1} P_{r+2} \cdots P_{m_{2 q+1}}$, so

$$
\alpha(x)=\sum \chi_{t s^{\prime} \cap a t^{\prime} s^{\prime}}(b x),
$$

where the summation is taken over $b \in Q_{n_{1}}, t \in T(C), t^{\prime} \in R$. We can write in a unique way $x=b^{\prime} z x^{\prime}$, where $x^{\prime} \in S^{\prime}, b^{\prime} \in Q_{n_{2}}, z \in R^{\prime}=P_{n_{1}+1} P_{n_{+}+2} \cdots P_{m_{2 q+1}}$. Since $Q_{n_{r}}$ is a group, we have

$$
\alpha(x)=\sum \chi_{t s^{\prime} \cap a t^{\prime} s^{\prime}}\left(b z x^{\prime}\right)
$$

where the summation is taken over $b \in Q_{n_{1}}, t \in T(C), t^{\prime} \in R$. We have

$$
\chi_{d S^{\prime} \cap a t^{\prime} s^{\prime}}\left(b z x^{\prime}\right)=1 \Leftrightarrow t=a t^{\prime}=b z \text {. }
$$

Since $T$ is a bijection, and $T^{-1}=T$, we have

$$
\chi_{t S^{\prime} \cap a t^{\prime} S^{\prime}}\left(b z x^{\prime}\right)=\chi_{T(t) S^{\prime} \cap T\left(a t^{\prime}\right) S}\left(T(b z) x^{\prime}\right) .
$$

It is clear that $T\left(a t^{\prime}\right)=a T^{\prime}\left(t^{\prime}\right)$ where $T^{\prime}$ is a bijection of $R$. It is also easy to see that $T(b z)=T^{\prime \prime}(b) z^{\prime}$ where $T^{\prime \prime}$ is a bijection of $Q_{n_{r}}$ and $z^{\prime} \in R^{\prime}$ is independent of b. So we have

$$
\alpha(x)=\sum \chi_{t s^{\prime} \cap a t^{\prime} s^{\prime}}\left(b z^{\prime} x^{\prime}\right)
$$

where the summation is taken over $t \in C, t^{\prime} \in R, b \in Q_{m_{r}}$ so

$$
\alpha(x)=\sum_{\substack{b \in Q_{m^{\prime}} \\ t \in C}} \chi_{t S^{\prime} \cap a S_{r}}\left(b z^{\prime} x^{\prime}\right)=\sum_{b \in Q_{m^{\prime}}} \chi_{Y_{2 q} \cap a S_{r}}\left(b z^{\prime} x^{\prime}\right) .
$$

From $\left(H_{2 q}\right)$ it follows that this quantity does not depend on $z^{\prime} x^{\prime}$, hence of $x$, which proves $\left(H_{2 q+1}\right)$ and concludes the proof.

ACKNOWLEDGement. The author thanks J. M. Rosenblatt for bringing this problem to his attention.

\section{REFERENCES}

1. F. P. Greenleaf, Invariant means on topological groups and their applications, Van Nostrand Math. Studies, vol. 16, Van Nostrand, Princeton, N. J., 1969.

2. L. A. Rubel and A. L. Shields, Invariant subspaces of $L^{\infty}$ and $H^{\infty}$, J. Reine Angew. Math. 272 (1975), 32-44.

Equite d'Analyse-Tour 46, Université Paris Vi, 4 Place Jussieu, 75230 Paris Cedex 05, France 